\title{
DiSTRIBUCIÓN, LOCALIZACIÓN E INHIBIDORES DE LAS POLIFENOL OXIDASAS EN FRUTOS Y VEGETALES USADOS COMO ALIMENTO
}

\author{
DISTRIBUTION, LOCATION AND INHIBITORS OF POLYPHENOL OXIDASES IN FRUITS AND VEGETABLES USED AS FOOD \\ ${ }^{\bullet}$ Jaime Morante Carriel$^{1}$, Anna Agnieszka Obrebska², Roque Bru-Martínez ${ }^{3}$, Mercedes Carranza Patiñoํㅡ, \\ Roberto Pico-Saltos ${ }^{4}$, Enrique Nieto Rodriguez ${ }^{1}$ \\ ${ }^{1}$ Grupo de Biotecnología y Biología Molecular, Facultad de Ciencias Ambientales, Universidad Técnica Estatal de Quevedo, \\ Av. Quito km, 1.5 vía a Santo Domingo de los Tsáchilas, EC-120501, Quevedo, Ecuador. jmorante@uteq.edu.ec, \\ mcarranza@uteq.edu.ec; jnieto@uteq.edu.ec \\ ${ }^{2}$ Grupo de microbiología y genética, Universidad de Alicante, España. anna.obrebska31@gmail.com \\ ${ }^{3}$ Grupo de Proteómica y Genómica Funcional de Plantas, Departamento de Agroquímica y Bioquímica, \\ Universidad de Alicante, España. roque.bru@ua.ec \\ ${ }^{4}$ Facultad de Ciencias de la Ingeniería, Universidad Técnica Estatal de Quevedo, Av. Quito km, 1.5 vía a Santo Domingo \\ de los Tsáchilas.EC-120501.Quevedo,Ecuador.rpico@uteq.edu.ec
}

\section{RESUMEN}

$\mathrm{L}$ as polifenol oxidasas (PPOs) son enzimas ubicuas que catalizan la reacción dependiente de oxígeno que transforma o-difenoles en o-quinonas. Estas quinonas son reactivas y capaces de modificar covalentemente un amplio abanico de especies nucleófilas, del interior de las células, que conduce a la formación de polímeros marrones, conocido como pardeamiento enzimático. El fenómeno de pardeamiento durante el crecimiento, recogida, almacenamiento y procesado de frutos $\mathrm{y}$ vegetales, es un problema de primera magnitud en la industria agroalimentaria y se reconoce como una de las principales causas de pérdidas de calidad y valor comercial. Produce cambios importantes tanto en la apariencia como en las propiedades organolépticas de frutos y vegetales comestibles, además suele ir asociado al desprendimiento de olores y efectos negativos sobre el valor nutricional. Aunque las PPOs se han descrito en diversos tejidos de plantas como raíces, semillas, hojas y frutos, el control de este fenómeno requiere un conocimiento bioquímico del tipo de sustratos fenólicos presentes en cada planta, el nivel de compuestos reductores, el nivel de accesibilidad del $\mathrm{O}_{2}$, de la naturaleza de los diferentes compuestos oxidables y de la polimerización y degradación de las o-quinonas. En este trabajo se presenta una revisión del efecto bioquímico, distribución, localización y posibles inhibidores de las PPOs en frutos y vegetales usados como alimento.

Palabras clave: Polifenol oxidasas, pardeamiento enzimático, frutos y vegetales.

Recibido: 10-junio-2013. Recibido en forma corregida: 7-septiembre-2013. Aceptado: 29-enero-2014.

Publicado como ARTÍCULO DE REVISION en Ciencia y Tecnología 7(1): 23-31

Enero-Junio de 2014

ISSN 1390-4051 impreso; ISSN 1390-4043 electrónico

(c) Ciencia y Tecnología. UTEQ. Quevedo-Ecuador
Abstract

$\mathrm{P}$ olyphenol oxidases (PPOs) are ubiquitous enzymes that catalyze the oxygen-dependent reaction that transforms o- diphenols to o-quinones. These quinones are reactive and capable of covalently modifying a wide variety of nucleophilic species into cells leading to the formation of brown polymers, known as enzymatic browning. The phenomenon of browning during growth, collection, storage and processing of fruits and vegetables, is a major problem in the food industry and is recognized as one of the leading causes of commercial value and quality loss, since it produces important changes in the appearance and organoleptic properties of edible fruits and vegetables, and it is often associated to the release of odors and negative effects on nutritional value. Although PPOs have been described in various plant tissues such as roots, sedes, leaves and fruits, the control of this phenomenon requires biochemical knowledge of the type of phenolic substrates present in each plant, the level of reducing compounds, the level of $\mathrm{O}_{2}$ accessibility, of the nature of the various oxidizable compounds and, finally, o-quinones polymerization and degradation. This paper present a review of the biochemical effect, distribution, location and potential inhitors of PPOs in fruits used for food.

Key words: Polyphenol oxidase, enzymatic browning, fruits and vegetables. 


\section{INTRODUCCIÓN}

$\mathrm{P}$ ara la mayoría de las especies frutales cuyo destino es el consumo en fresco, la calidad se basa en sus características intrínsecas, organolépticas y aspecto externo, sobre todo, en la forma, tamaño, color y ausencia de lesiones. El tamaño final que adquiere el fruto, la ausencia de magulladuras y de mancha púrpura, como desorden fisiológico, son aspectos valorados por el consumidor y, por tanto, son problemas importantes a los que se enfrentan los cultivadores en todo el mundo. Cualquiera de estos daños, cambian la textura o el color del tejido, debido a la destrucción de los compartimentos celulares del fruto, que permiten que los sustratos de naturaleza fenólica sean accesibles a la enzima polifenol oxidasa, dando lugar a polímeros oscuros (Sellés et al., 2007, Martínez et al., 2013). En general, todos los tipos de daños pueden resultar de una inapropiada manipulación o inadecuado embalaje. En el caso específico de pardeamiento por impacto, la incidencia de la severidad dependerá de la altura en que el fruto se deja caer y del tipo de superficie de impacto (Crisoto et al., 1993, 1996). Cualquier daño mecánico puede incrementar la tasa de respiración y producción de etileno lo que conlleva a una aceleración en la maduración, ablandamiento, pérdida de agua y deterioro general de los frutos (Crisoto et al., 1993). Además, el daño por impacto causa heridas superficiales en el fruto lo que facilita la entrada de microorganismos y el desarrollo de putrefacciones (Crisoto et al., 1996, Mishra y Gautam, 2013).

Las reacciones de oxidación que provocan el pardeamiento de frutos $y$ vegetales son de origen enzimático y están catalizadas principalmente por la enzima PPO, siendo su actividad particularmente alta en aquellos frutos y vegetales que contienen niveles altos de compuestos polifenólicos (Amiot et al., 1992), que va en detrimento del perfil nutricional del alimento (Vaughn y Duke, 1984; Mayer y Harel, 1991). Dado el impacto negativo de las PPOs en la industria alimenticia, se han realizado un gran número de publicaciones, sin embargo, su función en muchos vegetales no ha sido totalmente resuelta (Lee y Whitaker, 1995). Estas reacciones modifican las características organolépticas y nutricionales del alimento, depreciando su calidad (McEvily et al., 1992; Friedman, 1996; Matheis y Whitaker, 1984; Sánchez-Ferrer et al., 1995). La importancia de la PPO en el sector agroalimentario, ha atraído la atención de muchos investigadores desde los años sesenta hasta nuestros días, como se recoge en una revisión amplia sobre PPO (Mayer, 2006).

El fenómeno de pardeamiento de frutos y vegetales durante el crecimiento, recogida, almacenamiento y procesado, es un problema de primera magnitud en la industria alimentaria y se reconoce como una de las principales causas de pérdidas de calidad y valor comercial. Este pardeamiento produce cambios importantes tanto en la apariencia (colores oscuros) como en las propiedades organolépticas (sabor, textura) de vegetales comestibles (Mayer, 1987), y además suele ir asociado al desprendimiento de olores y efectos negativos sobre el valor nutricional (Amiot et al., 1992; Chen et al., 2000). Mientras que la lista de especies en las que se ha descrito la PPO ha ido creciendo de manera constate, la mayoría de los informes no añaden ningún detalle relevante sobre este tema. Por esta razón, se menciona algunos de los nuevos informes relacionados con la relevancia de las PPOs en frutos y vegetales usados como alimento.

En las plantas existe una gran cantidad y diversidad estructural de compuestos fenólicos pertenecientes a diversas familias como ácidos fenólicos, cumarinas, lignanos, ligninas y flavonoides (Lee y Whitaker, 1995; Tomás-Barberán y Espín, 2001). Estos compuestos desempeñan funciones importantes en las plantas, siendo las más relevantes las de protección frente a radiación ultravioleta y frente a condiciones de estrés biótico gracias a las propiedades antimicrobianas de los propios compuestos fenólicos y mediante el sellado de heridas por lignificación (Hermann, 1976; Ke y Salveit, 1988; Macheix et al., 1991).

La composición de fenoles en los tejidos vegetales varía considerablemente según la especie de que se trate, grado de madurez de los frutos y manejo post-cosecha de los mismos (Tomás-Barberán y Espín, 2001). Además, para una misma especie el contenido de fenoles es dependiente de la variedad (Ding et al., 1998b; Ding et al., 2001). En algunos frutos los niveles de fenoles aumentan a lo largo del desarrollo, alcanzándose los niveles más altos durante la recolección (Casado et al., 2003), mientras que para diferentes variedades el contenido de fenoles decrece hasta alcanzar el mínimo en el momento del cambio de color del fruto (envero) y después aumenta progresivamente alcanzando el máximo en la recolección. Sin embargo, las fluctuaciones en los niveles de fenoles suelen ser pequeños durante la maduración independientemente de algunas variedades (Ding et al., 1998b; Ding et al., 2001).

En la degradación oxidativa de estos compuestos fenólicos, participan dos enzimas que son muy relevantes en términos de calidad de frutos y vegetales, por la formación de melaninas que oscurecen los frutos. Estas enzimas son la polifenol oxidasa (PPO) y la peroxidasa (POD). A pesar de que las PODs están ampliamente distribuidas en el reino vegetal, su papel en el pardeamiento enzimático de frutos y vegetales esta 
todavía bajo discusión, debido a que el nivel de $\mathrm{H}_{2} \mathrm{O}_{2}$ interno en las plantas limita la actividad peroxidasa. Se ha propuesto que la $\mathrm{PPO}$ puede actuar como promotor de la POD puesto que en las reacciones de oxidación de compuestos fenólicos se genera $\mathrm{H}_{2} \mathrm{O}_{2}$ (Richard-Forget y Gauillard, 1997; Subramanian et al., 1999). El estado antioxidante de diferentes frutos y vegetales puede decrecer por la oxidación directa de estos en presencia de PPO y POD (Jiménez et al., 1998; Jiménez y GarcíaCarmona, 1999). Sin embargo, la principal enzima responsable del pardeamiento enzimático es la PPO, aunque no debe ser excluido un posible efecto sinérgico entre PPO y POD (Tomás-Barberán y Espín, 2001).

$\mathrm{La}$ prevención y control del pardeamiento enzimático, requiere un conocimiento bioquímico del tipo de sustratos fenólicos presentes en cada planta, del nivel de compuestos reductores, el nivel de accesibilidad del $\mathrm{O}_{2}$, la naturaleza de los diferentes compuestos oxidables y la polimerización y degradación de las o-quinonas. Además, es necesario conocer el nivel de PPO y los sustratos disponibles a lo largo de los diferentes estados de desarrollo de la planta y sobre todo, es importante distinguir entre el pardeamiento enzimático y el no enzimático (figura 1) (Lee y Whitaker, 1995).

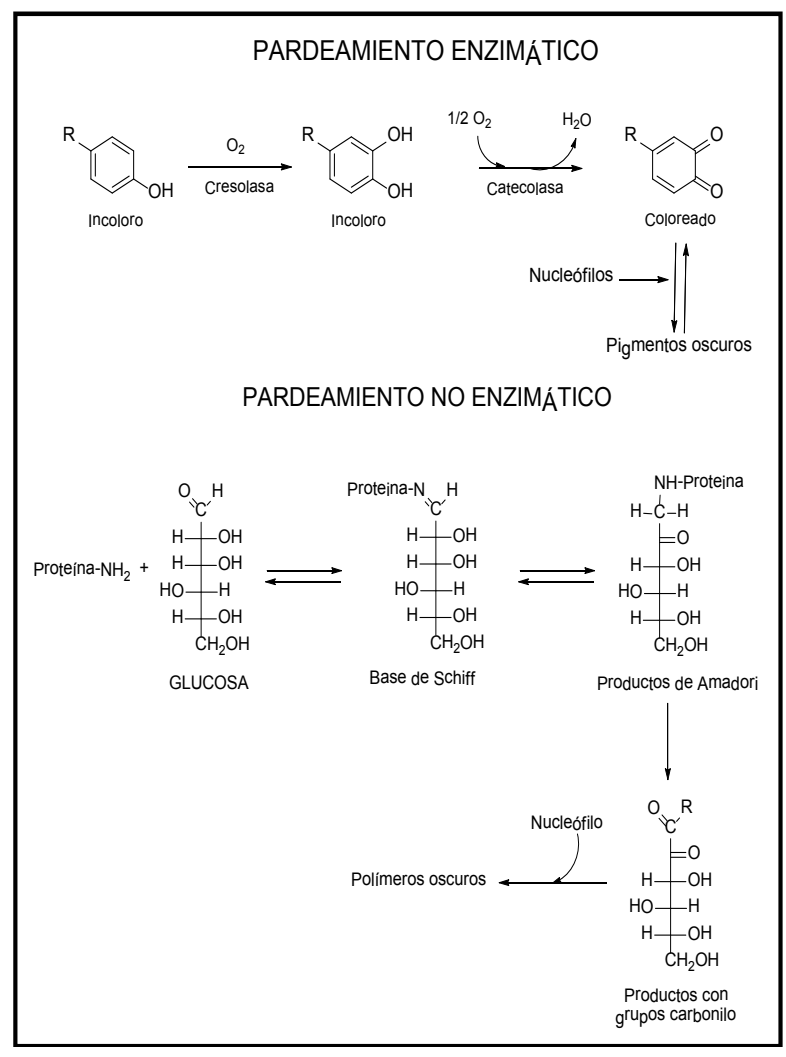

Figura 1. Reacciones de pardeamiento enzimático y no enzimático (Reacción de Maillard)
El pardeamiento no enzimático consiste en la condensación de un grupo aldehído o cetona de un azúcar con un grupo amino libre para formar una base de Schiff, la cual se reorganiza para formar una cetamina estable (producto de Amadori) y finalmente se degradan a productos reactivos que contienen grupos carbonilo. Estos grupos pueden reaccionar con grupos amino dando lugar a polímeros oscuros. Esta reacción que tiene lugar al calentar mezclas de aminoácidos y carbohidratos (Walker y Mckersie, 1993) se ha descrito en uva (Cheynier y Ricardo da Silva, 1991) y manzana (Oleszek et al., 1989; Richard-Forget et al., 1992a).

Entre las diferentes técnicas para controlar el pardeamiento y mantener la calidad de frutos y vegetales, una de las más usadas es la aplicación de inhibidores químicos, los cuales consiguen inactivar los mecanismos no deseados. La actividad de esta clase de inhibidores implica una interacción directa con la enzima o reaccionan preferiblemente con el producto que conduce por reacción no enzimática a la formación de pigmentos oscuros.

\section{Distribución y localización de PPOs}

Las polifenol oxidasa (PPOs), son metaloenzimas ampliamente distribuidas en la escala filogenética, encontrándose tanto en organismos procariotas como en eucariotas. Se trata de una enzima detectada en algas, briófitos, pteridófitos, gimnospermas y angiospermas (Mayer y Harel, 1979).

La polifenol oxidasa se ha descrito en diversos tejidos de plantas como raíces (Pérez-Gilabert et al., 2001; Gandía-Herrero et al., 2004), semillas (Paul y Gowda, 2000), hojas (Robinson y Dry, 1992; SánchezFerrer et al., 1993; Chazarra et al., 1996; Mazzafera y Robinson, 2000; Chazarra et al., 2001; Shi et al., 2002) y frutos (Fraignier et al., 1995; Murata et al., 1992; Ding et al., 1998a; Serradell et al., 2000; Casado et al., 2005, Sellés et al., 2006; 2007).

Estudios clásicos de PPO localizan a la enzima en la fracción soluble de las células o fuertemente unida a membranas subcelulares (Mayer y Harel, 1979). Sin embargo, esta asignación de localización se debe en algunos casos al método de extracción utilizado. A pesar de los posibles artefactos la mayoría de las polifenol oxidasas vegetales se encuentran asociadas a membranas, principalmente a los tilacoides del cloroplasto (Tolbert, 1973). El tipo de unión a la membrana depende del tejido y del estado de desarrollo de la planta. Aunque las PPOs se han localizado en los tilacoides (Golbeck y Cammarata, 1981; Chazarra et al., 1996), no son proteínas intrínsecas de membrana. A pesar de que en el tabaco (Hofer, 1964) se libera simplemente por fuerza iónica débil, para la mayoría de los tejidos hacen falta tratamientos más enérgicos 
para su solubilización: detergentes como Tritón X-100, (Harel y Mayer, 1971), Tritón X-114 (Sánchez-Ferrer et al., 1989), enzimas proteolíticas (Mayer, 1966), etc. Algunos tratamientos pueden alterar la estructura y la conformación de la enzima provocando el paso de su forma inactiva o latente a su forma activa (Kenten, 1958), si bien la recuperación de la enzima nativa latente es posible mediante el uso de detergentes no iónicos suaves (Sánchez-Ferrer et al,, 1994). Esta solubilización también tiene lugar en condiciones naturales, como son la maduración de las frutas y el envejecimiento de los tejidos (Harel et al., 1966; Kidron et al., 1978).

Diversas evidencias apuntan hacia la localización concreta de las PPOs de plantas superiores en las membranas tilacoidales (Mayer, 1987; Vaughn et al., 1988; Kowalski et al., 1992), aunque existen trabajos que describen la existencia de algunas PPOs vegetales en forma soluble en el lumen de los tilacoides (Sommer et al., 1994; Murata et al., 1997).

Varios trabajos en los que se utilizan frutos como fuente de PPO (Casado et al., 2005; Ding et al., 1998a; Gandía-Herrero et al., 2005a y b; Sellés et al. 2006), han descrito la presencia de actividad PPO en fracciones particuladas y solubles del mismo tejido, mostrando propiedades cinéticas diferentes, que comprometen la localización exclusiva de la proteína en los plastos. Técnicas de inmunodetección con oro coloidal han permitido demostrar la localización de PPO en aquellos tejidos con escasa o nula presencia de cloroplastos en células del parénquima denominadas idioblastos, que además acumulan cantidades elevadas de fenoles (Thipyapong y Steffens, 1997).

Las PPOs vegetales son proteínas codificadas por el núcleo en forma de precursor (Lax et al., 1984; Kowalski et al., 1990). El análisis de las secuencias de aminoácidos a partir de cDNA de PPO de tomate (Newman et al., 1993; Hunt et al., 1993), haba (Cary et al., 1992) y espinaca (Hind et al., 1995) muestran que la enzima PPO presenta un doble péptido señal de transporte al estroma y tilacoides (Keegstra y Froehlich, 1999, Keegstra y Cline, 1999).

Estudios realizados por Sommer et al. (1994) con cloroplastos aislados, demuestran que el proceso de translocación por transporte al cloroplasto de los polipéptidos de PPO de tomate $(67 \mathrm{kDa})$ resultantes de la traducción en el citosol se lleva a cabo en dos pasos. Un primer paso implica la translocación por transporte hacia el estroma, produciéndose la eliminación del péptido de tránsito $\mathrm{N}$-terminal y proporcionando un intermediario de $62 \mathrm{kDa}$ y posteriormente un segundo paso de translocación por transporte a los tilacoides por un proceso dependiente de ATP, que genera una proteína madura de $59 \mathrm{kDa}$. En este tránsito el péptido señal se elimina por una peptidasa cloroplastídica
(Koussevitzky et al., 1998). Así mismo, Koussevitzky et al. (2004) demostraron que el pretratamiento con metil jasmonato (MeJA) de plantas de tomate incrementa considerablemente la eficiencia de la translocación por transporte de la polifenol oxidasa precursora (pPPO) del plástido al tilacoide. Estos autores muestran un incremento en el nivel de la polifenol oxidasa madura (mPPO) en la fracción del tilacoide después de las 8-16 h del tratamiento. Además este aumento en el nivel de mPPO parece ser específico del tratamiento con MeJA para las plantas de tomate, debido a que otros tratamientos que incrementan la expresión de genes de PPO como daños mecánicos o exposición de las plantas a etileno durante 48 horas (Thipyapong y Steffens, 1997), no muestran ningún efecto en la translocación por transporte del pPPO hacia el tilacoide. Esta eficiencia observada en el transporte de pPPO para las plantas de tomate, se ha descrito para otras plantas de la misma familia como el tabaco, mientras que el tratamiento con MeJA de plantas leguminosas como el guisante, no afectó al transporte de los dos precursores de PPO (Koussevitzky et al., 2004). La translocación de PPO está fuertemente inhibida por la presencia de pequeñas concentraciones de $\mathrm{Cu}^{2+}$, lo que sugiere que la proteína ha de transportarse en su forma desplegada y además, indica que el dominio de unión a cobre tiene una fuerte avidez por este metal (Sommer et al., 1994). A pesar de esta unión del sitio activo por el cobre, los procesos de homogenización y extracción de tejidos para realizar los estudios de actividad de PPO en vegetales pueden provocar la pérdida de este metal de su lugar correspondiente en la enzima, induciendo la pérdida de actividad que a veces se puede recuperar mediante incubación con sales de cobre (Mari et al., 1998; Casado et al., 2005). Nielsen et al. (1996) descubrieron que no hay péptido de tránsito $\mathrm{N}$-terminal en tirosinasas de hongos, lo cual es coherente con la ausencia de compartimentos plastídicos en estos organismos. Así pues, las tirosinasas de hongos se encuentran localizadas en el citosol (Wickers et al., 1995).

\section{Inhidibores de PPOs}

Entre los diversos tipos de inhibidores de PPOs se destacan cuatro grupos: sulfitos, agentes antioxidantes o reductores, acidulantes y compuestos quelantes. Los sulfitos son los compuestos más efectivos en prevenir el pardeamiento enzimático (Sapers, 1993). Aunque el mecanismo de actuación de los sulfitos para prevenir el pardeamiento no está claro, pueden provocar una inhibición directa de la enzima, como se ha observado en la inhibición de PPO de fresa por metabisulfito de sodio (Wesche-Ebeling y Montgomery, 1983), pueden interaccionar con los intermedios evitando que estos participen en la formación de pigmentos 
(Sayavedra-Soto y Montgomery, 1986) o pueden actuar como agentes reductores convirtiendo a las quinonas en difenoles (Valero et al., 1988). A pesar de su efectividad en la prevención de la calidad de frutos y vegetales, estos compuestos están sujetos a restricciones debido a que provocan efectos adversos en la salud en personas sensibles. Entre los antioxidantes, se han empleado compuestos fenólicos sintéticos como butilhidroxitolueno (BHT) y butilhidroxianisol (BHA), ampliamente empleados en alimentación para proteger el sabor y color de los alimentos y algunos compuestos fenólicos naturales como tocoferol, derivados del ácido cinámico y flavonoides como la quercetina y el kaemferol (Ashie et al., 1996).

Una de las mejores alternativas al uso de los sulfitos es el ácido ascórbico (Wang et al., 2013), este compuesto es altamente efectivo en la inhibición del pardeamiento por su habilidad de reducir las quinonas producidas por la PPO a los fenoles antes de que la reacción de formación de pigmentos tenga lugar. Sin embargo, el ácido ascórbico es muy reactivo y se oxida rápidamente a ácido dehidroascórbico (DHAA), pudiendo reaccionar con otros compuestos que conllevan a cambios en la calidad de los frutos. A veces se utiliza en combinación con acidulantes, siendo el más utilizado el ácido cítrico debido a su presencia natural en tejidos. Otros inhibidores son los compuestos sulfhidrilos como mercaptoetanol, ditiotreitol y tiourea por su habilidad como agentes reductores, sin embargo, las concentraciones necesarias para prevenir el deterioro del fruto no son permitidas en alimentación. La cisteína se ha mostrado como un inhibidor fuerte de PPO en banana y manzana, siendo incluso más efectivo que el metabisulfito (Ashie et al., 1996; Richard-Forget et al., 1992b; Valero et al., 1988), sin embargo, la concentración necesaria para alcanzar altos niveles de inhibición tiene efectos negativos en el sabor de los frutos. Además se han empleado agentes quelantes como ácidos policarboxílicos, polifosfatos y ácido etilen-diamino-tetraacético (EDTA) para inactivar a la PPO. A pesar de que muchos de estos compuestos son bastante efectivos en el control del pardeamiento enzimático, a menudo su uso en alimentación está limitado por producir efectos adversos en la salud, debido a un coste efectivo o porque su acción es sólo temporal como el ácido ascórbico. Por este motivo, cada vez se recurre más a la utilización de productos naturales como las ciclodextrinas, que tienen la capacidad de incluir una amplia variedad de moléculas orgánicas e inorgánicas, incluyendo a los polifenoles (Cai et al., 1990; Bru et al., 1995), dentro de su cavidad hidrofóbica, aunque el coste es elevado. Recientemente, se han utilizado productos de la reacción de Maillard, sintetizados a partir de azúcares (pentosa, hexosa, o disacárido) y compuestos tiol como inhibidores del pardeamiento enzimático en frutos (manzana) y vegetales (champiñón y berenjena) crudos y procesados (Billaud et al., 2005).

\section{Conclusiones}

T a presencia de PPO se ha podido determinar y caracterizar empleando hojas y frutos de numerosas especies vegetales como fuente enzimática, según esta caracterizacion, los niveles de PPO varían dependiendo de la especie, cultivar, estadio de maduración y estadio fenológico. En tejidos vegetales intactos las PPOs y sus sustratos fenólicos permanecen en compartimentos separados (cloroplastos y vacuolas), por lo que no tiene lugar ninguna reacción. La desorganización de la integridad de las células sucede como consecuencia de daños mecánicos o de forma natural durante procesos de senescencia, provocando una ruptura celular y una puesta en contacto de PPO y fenoles dando lugar a reacciones de pardeamiento enzimático observadas en frutos maduros, tejidos dañados, procesados y en tejidos afectados por fisiopatías. El conocimiento de las PPOs en frutos y vegetales, es un paso fundamental en la investigación, puesto que permitirá mejorar la calidad y apriencia de los frutos y vegetales frescos y procesados, reduciendo las pérdidas económicas que supone su efecto en la industria agroalimentaria.

\section{Agradecimiento}

Los autores agradecen al Grupo de Investigación Proteómica y Genómica Funcional de Plantas de la Universidad de Alicante, España. Al Grupo de Biotecnología y Biología Molecular de la Universidad Técnica Estatal de Quevedo, Ecuador y a la Secretaría Nacional de Educación Superior, Ciencia, Tecnología e Innovación (Senescyt) del Gobierno de Ecuador por su contribución y apoyo.

\section{Bibliografía}

Amiot, M., Tacchini, M., Aubert, S. and Nicolas, J. 1992. Phenolic composition and browning susceptibility of various apple cultivars at maturity. Journal of Food Science 57(4): 958-962.

Ashie, I.N.A., Simpson, B.K. and Smith, J.P. 1996. Critical Reviews in Food and Science Nutrition 36(1-2): 1-30.

Billaud, C., Maraschin, C.Y., Chow, N., Chériot, S., Peyrat-Maillard, M.N. and Nicolas, J. 2005. Maillard reaction products as natural antibrowning agents in fruit and vegetable technology. Molecular Nutrition and Food Research 49(7): 656-662. 
Bru, R., Sánchez-Ferrer, A., Pérez- Gilabert, M., LópezNicolás, J. and García-Carmona, F. 1995. Plant protein purification using cloud point extraction (CPE). In: Surfactants in solution, Chattopadhyay, A. and Mittal, K. (Eds). Marcel Dekker, Inc. New York, 367-377. New York.

Cai, Y., Caffney, H.G., Lilley, T.H., Magnolato, D., Martin, R., Spencer, C.M. and Haslam, E. 1990. Polyphenol interactions. Part 4. Model studies with caffeine and cyclodextrins. Journal of the Chemical Society, Perkin Transaction, 2:2197-2209.

Cary, J.W., Lax, A.R. and Flurkey, W.H. 1992. Cloning and characterization of cDNAs coding for Vicia faba polyphenol oxidase. Plant Molecular Biology, 20:245-253.

Casado-Vela, J., Sellés, S., Gómez-Lucas, I. and Bru, R. 2003. Evolution of phenolics and polyphenol oxidase isoenzymes in relation to physical-chemical parameters during loquat (Eriobotrya japonica cv. Algerie) fruit developement and ripening. Options Mediterranees, Serie A: Seminaires Mediterranees, 58:161-164.

Casado-Vela, J., Sellés, S., and Bru, R. 2005. Proteomic approach to blossom-end rot in tomato fruits (Lycopersicon esculenrum M.). Proteomics 5(10): 2488-2496.

Chazarra, S., Cabanes, J., Escribano, J. and GarcíaCarmona, F. 1996. Partial purification and characterization of latent polyphenol oxidase in iceberg Lettuce (Latuca sativa L.). Journal of Agricultural and Food Chemistry 44:984-988.

Chazarra, S., García-Carmona, F. and Cabanes, J. 2001. Evidence for a tetrameric form of iceberg lettuce (Latuca sativa) Polyphenol oxidase: purification and characterization. Journal of Agricultural and Food Chemistry 49: 4870-4875.

Chen, L., Berenbaum, M., Zangerl, A. and Engeseth, N. 2000. Honeys from different floral sources as inhibitors of enzymatic browning in fruit and vegetable homogenates. Journal of Agricultural and Food Chemistry 48: 4997-5000.

Cheynier, V. and Ricardo da Silva, J.M. 1991. Oxidation of grape procyanidins in model solutions containing trans-caffeoyltartaric acid and polyphenol oxidase. Journal of Agricultural and Food Chemistry 39:1047-1049.

Crisoto, C., Andris, H. and Mitcham, B. 1993. Tips to reduce bruising during apple harvesting. Central Valley Postharvest Newsletter 2(2): 6-10.

Crisoto, C., Garner, D., Ritenour, M., Schap, J. and Rushing, J. 1996. Developing critical bruising threshold for stone fruit. Central Valley Postharvest Newsletter 5(1): 11-13.
Ding, C.K., Chachin, K., Ueda, Y. and Imahori, Y. 1998a. Purification and properties of polyphenol oxidase from Loquat fruit. Journal of Agricultural and Food Chemistry 46(10): 4144-4149.

Ding, C.K., Chachin, K., Ueda, Y. and Mochioka, R. 1998b. Changes in polyphenol concentrations and polyphenol oxidase activity of loquat (Eriobotrya japonica Lindl.) fruits in relation to browning. Journal of the Japanese Society for Horticultural Science 67(3): 360-366.

Ding, C.K., Chachin, K., Ueda, Y., Imahori, Y. and Wang, C.Y. 2001. Metabolism of phenolic compounds during loquat fruit developement. Journal of Agricultural and Food Chemistry 49(6): 2883-2888.

Fraignier, N., Marqués, L., Fleuriet, A. and Macheix, J.J. 1995. Biochemical and inmunochemical characteristics of polyfenol oxidases from differents fruits of Prunus. Journal of Agricultural and Food Chemistry 43: 2375-2380.

Friedman, M. 1996. Food Browning and its prevention: an overview. Journal of Agricultural and Food Chemistry 45: 1091-1096.

Gandía-Herrero, F., García-Carmona, F. and Escribano, J. 2004. Purification and characterization of a latent polyphenol oxidase from beet root (Beta vulgaris L.). Journal of Agricultural and Food Chemistry 52(3): 606-615.

Gandía-Herrero, F., Jiménez-Atiénzar, M., Cabanes, J., García-Carmona, F. and Escribano, J. 2005a. Differential activation of a latent polyphenol oxidase mediated by sodium dodecyl sulfate. Journal of Agricultural and Food Chemistry 53(17): 6825-6830.

Gandía-Herrero, F., Jiménez-Atiénzar, M., Cabanes, J., García-Carmona, F. and Escribano, J. 2005b. Evidence for a common regulation in the activation of a polyphenol oxidase by trypsin and sodium dodecyl sulfate. Biological Chemistry 386(6): 601607.

Golbeck, J.H. and Cammarata, K.V. 1981. Spinach thylakoid polyphenol oxidase. Isolation, activation and properties of the native chloroplast enzyme. Plant Physiology 67(5): 977-984.

Harel, E., Mayer, A.M. and Shain, Y. 1966. Catechol oxidases, endogenous substrates and browning in developing apples. Journal of the Science of Food and Agriculture 17(9): 389-392.

Harel, E. and Mayer, A.M. 1971. Partial purification and properties of catechol oxidases in grapes. Phytochemistry 10(1): 17-22.

Hermann, K. 1976. Flavonols and flavones in food plants: a review. Journal of Food Science and Technology 11(5): 433-448. 
Hind, G., Marsshak, D. and Coughlan, S. 1995. Spinach thylakoid polyphenol oxidase: cloning, characterization, and relation to a putative protein kinase. Biochemistry 34(25): 8157-8164.

Hofer, A. 1964. Intracellular localization of phenolases in tobacco leaves. Planta 62: 137-159.

Hunt, M.D., Eannetta, N.T., Haifeng, Y., Newman, S.M. and Steffens, J.C. 1993. cDNA cloning and expression of potato polyphenol oxidase. Plant Molecular Biology 21: 59-68.

Jiménez, M., Escribano-Cebrián, J. and GarcíaCarmona, F. 1998. Oxidation of the flavonol fisetin by polyphenol oxidase. Biochimica et Biophysica Acta 1425(3): 534-542.

Jiménez, M. and García-Carmona, F. 1999. Myricetin, an antioxidant flavonol, is a substrate of poliphenol oxidase. Journal of the Science of Food and Agriculture 79(14): 1993-2000.

Ke, D. and Salveit, E. 1988. Plant hormone interaction and phenolic metabolism in the regulation of russet spotting in iceberg lettuce. Plant Physiology 88(4): 1136-1140.

Keegstra, K. and Cline, K. 1999. Protein import and routing systems of chloroplasts. Plant Cell 11(4): 557-570.

Keegstra, K. and Froehlich, J.E. 1999. Protein import to chloroplasts. Curr. Opin. Plant Biology 2(6): 471476.

Kenten, R.H. 1958. Latent phenolase in extracts of broad bean (Vicia faba L.) leaves. Activation by anionic weyying agents. Biochemical Journal 68(2): 244-251.

Kidron, M., Harel, E. and Mayer, A.M. 1978. Catechol oxidase activity in grapes and wine. American Journal of Enology and Viticulture 29: 30-35.

Koussevitzky, S., Ne'eman, E., Sommer, A., Steffens, J. and Harel, E. 1998. Purification and properties of a novel chloroplast stromal peptidase. Journal of Biological Chemistry 273(42): 27064-27069.

Koussevitzky, S., Ne'eman, E. and Harel, E. 2004. Import of polyphenol oxidase by chloroplasts is enhanced by methyl jasmonate. Planta 219: 412419.

Kowalski, S., Banberg, J., Tingey, W. and Steffens, J. 1990. Insect resistance in the wild potato Solanum berthaultii: Inheritance of granular trichome polyphenol oxidase. Journal of Heredity 81(6): 475-478.

Kowalski, S.P., Eannetta, N.T., Hirzal, A.T. and Steffens, J.C. 1992. Purification and characterisation of polyphenol oxidase from glandular trichomes of Solanum berthaultii. Plant Physiology 100(2): 677-684.
Lax, A.R., Vaughn, K.C. and Templeton, G.E. 1984. Nuclear inheritance of polyphenol oxidase in nicotiana. Journal of Heredity 75(4): 285-287.

Lee, C.Y. and Whitaker, J. 1995. Enzymatic browning and its prevention, ACS Symposum Series 600, Washington, D.C; American Chemical society Ed.

Macheix, J.J., Sapis, J.C. and Fleuriet, A. 1991. Phenolic compounds and polyphenol oxidase in relation to browning in grapes and wines. Critical Reviews in Food Science and Nutritien 30(4): 441-486.

Mari, S., Marqués, L., Breton, F., Karamanos, Y. and Macheix, J.J. 1998. Unfolding and refolding of active apple polyphenol oxidase. Phytochemistry 49(5): 1213-1217.

Martínez-Márquez, A., Morante-Carriel, J., SellésMarchart, S., Martínez-Esteso, M.J., PinedaLucas, J.L., Luque I. and Bru-Martínez, R. 2013. Development and Validation of MRM Methods to Quantify Protein Isoforms of Polyphenol Oxidase in Loquat Fruits. J. Proteome Res. 12: 5709-5722

Matheis, G. and Whitaker, J.R. 1984. Modification of proteins by polyphenol oxidase and peroxidase and their products, Journal of Food Biochemistry 8(3): 137-162.

Mayer, A.M. 1966. Catechol oxidase; enzymic liberation from sugar beet chloroplasts. Phytochemistry 5(6): 1297-1301.

Mayer, A. M. and Harel, E. 1979. Polyphenol oxidases in plants. Phytochemistry 18(2): 193-215.

Mayer, A.M. 1987. Polyphenol oxidases in plants-recent progress. Phytochemistry 26(1): 11-20

Mayer, A.M. and Harel, E. 1991. Polyphenoloxidases and their significance in fruits and vegetables. In Food enzymology; Fox, P. F., Ed.; Elsevier Applied Science: London, U.K., pp. 373-398.

Mayer, A.M. 2006. Polyphenol oxidases in plants and fungi: Going places? A review. Phytochemistry, 67(21): 2318-2331.

Mazzafera, P. and Robinson, S.P. 2000. Characterization of polyphenol oxidase in coffee. Phytochemistry, 55(4): 285-296.

McEvily, A., Iyengar, R. and Otwell, S. 1992. Inhibition of Enzymatic Browning in Foods and Beverages. Critical Review in Food Science and Nutrition, 32(3): 253-273.

Mishra, B.B., Gautam, S., Sharma, A. 2013. Free phenolics and polyphenol oxidase (PPO): the factors affecting post-cut browning in eggplant (Solanum melongena). Food Chem. 139(1-4): 105-114

Murata, M., Kurokami, C. and Homma, S. 1992. Purification and some properties of chlorogenic acid oxidase from apple (Malus pumila). Bioscience Biotechnology and Biochemistry 56(11): 17051710 . 
Murata, M., Tsurutani, M., Hagiwara, S. and Homma, S. 1997. Subcellular location of polyphenol oxidase in apples. Bioscience Biotechnology and Biochemistry 61(9): 1495-1499.

Newman, S.M., Eannetta, N.T., Yu, H., Prince, J.P., de Vincente, M.C., Anksley, S.D. and Steffens, J.C. 1993. Organisation of the tomato polyphenol oxidase gene family. Plant Molecular Biology 21: 1035-1051.

Nielsen, H., Engelbrecht, J., Von Heijne, G. and Brunak, S. 1996. Defining a similarity threshold for a functional protein sequence pattern: The signal peptide cleavage site. Proteins-Structure, Function and Genetics 24: 165-177.

Oleszek, W., Chang, Y.L., Jaworski, A.W. and Price, K.R. 1989. Apple phenolics and their contribution to enzymatic browning reactions. Acta Societatis Botanicorum Poloniae 58(2): 273-283.

Paul, B. and Gowda, L.R. 2000. Purification and characterization of a polyphenol oxidase from the seeds of Field bean (Dolichos lablab). Journal of Agricultural and Food Chemistry 48(9): 38393846.

Pérez-Gilabert, M., Morte, A., Honrubia, M. and García-Carmona, F. 2001. Partial purification, characterization, and histochemical localization of fully latent desert truffle (Terfezia Claveryi Chatin) polyphenol oxidase. Journal of Agricultural and Food Chemistry 49(4): 1922-1927.

Richard-Forget, F.C., Goupy, P.M. and Nicolas, J.J. 1992a. Cysteine as an inhibitor of enzymatic browning. Kinetic studies. Journal of Agricultural and Food Chemistry 40: 2108-2113.

Richard-Forget, F.C., Rouet-Mayer, M.D., Goupy, P.M., Philippon, J. and Nicolas, J.J. 1992b. Oxidation of chlorogenic acid, catechins and 4-methylcatechol in model solutions by apple polyphenol oxidase. Journal of Agricultural and Food Chemistry 40: 2114-2122.

Richard-Forget, F.C. and Gauillard, F. 1997. Oxidation of chlorogenic acid, catechins and 4-methylcatechol in model solutions by combination of pear (Pyrus communis cv. Williams) polyphenol oxidase and peroxidase: a possible involvement of peroxidase in enzymatic browning. Journal of Agricultural and Food Chemistry 45(7): 2472-2476.

Robinson, S.P. and Dry, I.B. 1992. Broad bean leaf polyphenol oxidase is a 60-kilodalton protein susceptible to proteolytic cleavage. Plant Physiology 99(1): 317-323.

Sánchez-Ferrer, A., Bru, R. and García-Carmona, F. 1989. Novel procedure for extraction of a latent grape polyphenol oxidase using temperatureinduced phase separation in Triton X-114. Plant
Physiology 91(4): 1481-1487.

Sánchez-Ferrer, A., Laveda, F. and García-Carmona, F. 1993. Substrate-Dependent activation of latent potato leaf polyphenol oxidase by anionic surfactants. Journal of Agricultural and Food Chemistry 41: 1583-1586.

Sánchez-Ferrer, A., Bru, R. and García-Carmona, F. 1994. Phase separation of biomolecules in polyoxyethylene glycol nonionic detergents. Critical Reviews in Biochemistry and Molecular Biology 29(4): 275-313.

Sánchez-Ferrer, A., Rodríguez-Lopez, J.N., GarcíaCasanovas, F. and García-Carmona, F. 1995. Tyrosinase: a comprensive review of its mechanism. Biochimica et Biophysica Acta 1247: 1-11.

Sapers, G.M. 1993. Browning of foods: control by sulfites, antioxidants and other means. Food Thecnology 47: 75-84.

Sayavedra-Soto, T. and Montgomery, M. 1986. Inhibition of polyphenol oxidase by sulfite. Journal of Food Science 51: 1531-1536.

Sellés-Marchart, S., Casado-Vela, J., and Bru-Martínez, R. 2006. Isolation of a latent polyphenol oxidase from loquat fruit (Eriobotrya japonica Lindl.): kinetic characterization and comparison with the active form. Archives of Biochemistry and Biophysics 446(2): 175-85.

Sellés-Marchart, S., Casado-Vela, J., and BruMartínez, R. 2007. Effect of detergents, trypsin and unsaturated fatty acids on latent loquat fruit polyphenol oxidase: basis for the enzyme's activity regulation. Archives of Biochemistry and Biophysics 464(2): 295-305.

Serradell, M.A., Rozenfeld, P.A., Martínez, G.A., Civello, P.M., Chaves, A.R. and Añon, M.C. 2000. Polyphenol oxidase activity from strawberry fruit (Fragaria ananassa, Duch, cv Selva): characterisation and partial purification. Journal of the Science of Food and Agriculture 80(9): 14211427.

Shi, C., Dai, Y., Xu, X., Xie, Y. and Liu, Q. 2002. The purification of polyphenol oxidase from tobacco. Protein Expression and Purification 24(1): 51-55.

Sommer, A., Ne'eman, E., Steffens, J., Mayer, A.M. and Harel, E. 1994. Import, targeting and processing of a plant polyphenol oxidase. Plant Physiology 105(4): 1301-1311.

Subramanian, N., Venkatesh, P. Ganguli, S. and Sinkar, V.P. 1999. Role of polyphenol oxidase and peroxidase in the generation of black tea theaflavins. Journal of Agricultural and Food Chemistry 47(7): 2571-2578.

Thipyapong, P. and Steffens, J. 1997. Tomato polyphenol oxidase. Differential response of the polyphenol 
oxidase F promoter to injuries and wound signal. Plant Physiology 115(2): 409-418.

Tolbert, N. 1973. Activation of polyphenol oxidase of chloroplasts. Plant Physiology 51(2): 234-244.

Tomás-Barberán, F.A. and Espín, J.C. 2001. Phenolic compounds and related enzymes of quality in fruits and vegetables. Journal of the Science of Food and Agriculture 81(9): 853-876.

Valero, E., Escribano, J. and García-Carmona, F. 1988. Reactions of 4-methyl-o-benzoquinone generated chemically or enzymically, in the presence of L-proline. Phytochemistry 27: 2055-2061.

Vaughn, K.C., and Duke, S.O. 1984. Function of polyphenol oxidase in higher plants. Physiologia Plantarum 60(1): 106-112.

Vaughn, K.C., Lax, A.R and Duke, S.O. 1988. Polyphenol oxidase: the chloroplast oxidase with no established function. Physiologia Plantarum 72(3): 659-665.
Walker, M. and McKersie, B. 1993. Role of the ascorbate-glutathione antioxidant system in chilling resistance of tomato. Journal of Pant Physiology 141(2): 234-239.

Wang, C., Jing-fang, Z., Zhang, Y., and Cheng, B. 2013. Characterization and Inhibitors of Polyphenol Oxidase from Chinese Toon. Food Biotechnology 27(3): 165-178

Wesche-Ebeling, P. and Montgomery, M.W. 1983. Extraction and partial characterization of strawberry polyphenol oxidase. IFT Meet. , Abstr. 43rd Annu 295.

Wickers, H.J., Van de Bosch, T., Gerritsen, Y.A.M., Oyevaar, J.I., Ebbelaar, M.C.E., Recourt, K. and Kerrigan, R.W. 1995. Mushroom Science. XIV 720. 